\title{
Vection depends on perceived surface properties
}

\author{
Juno Kim $^{1}$ • Sieu Khuu ${ }^{1}$ - Stephen Palmisano ${ }^{2}$
}

Published online: 7 March 2016

(C) The Psychonomic Society, Inc. 2016

\begin{abstract}
Optic flow provides important information for the perception of self-motion and can be generated by both diffuse and specular reflectance. Previous self-motion research using virtual environments has primarily considered the properties of diffuse optic flow, but not of specular flow. We used graphical simulations to examine the extent to which visually induced self-motion (vection) is robust against the variations in optic flow generated by different surface optics. We found that specular flow alone was capable of generating vection that was equivalent in strength to that generated by diffuse flow (Exp. 1). To test whether this specularly induced vection depends on midlevel visual processing, we measured vection strengths under conditions in which the luminance polarity of specular highlights was inverted. We found that inverting the luminance of specular reflections impaired vection strength, as compared with the vection generated by conditions with ecologically correct diffuse and/or specular flow (Exp. 2). We also found these variations in vection strength were correlated with the perceived relief heights of the surfaces depicted in the image sequences. These findings together suggest that vection can be induced by pure specular flow and that it requires processing beyond the computation of retinal motion velocities - most likely, processes involved in the recovery of 3-D surface shape.
\end{abstract}

Juno Kim

juno.kim@unsw.edu.au

1 School of Optometry and Vision Science, University of New South Wales, Sydney, NSW 2052, Australia

2 School of Psychology, University of Wollongong, Wollongong, NSW, Australia
Keywords Self-motion perception · Optic flow · Surface and material properties $\cdot 3 \mathrm{D}$ shape perception $\cdot$ Vection

Optic flow is caused by the way that patterns of light, reflected by surrounding objects and surfaces toward our eyes, continually change as we move through the environment (Gibson, 1966). Visual percepts of self-motion depend on the visual system's ability to compute heading and velocity in threedimensional (3-D) space from these two-dimensional (2-D) patterns of optic flow (see Lappe, Bremmer, \& van den Berg, 1999, for a review). The importance of optic flow for selfmotion perception is evident in the fact that highly compelling illusions of self-motion can be induced in stationary observers by visual stimulation alone, traditionally known as vection (Dichgans \& Brandt, 1978; for alternative definitions of this term, see Palmisano, Allison, Schira, \& Barry, 2015). Most vection studies have used rather schematic dot motion displays to induce these illusions of self-motion. However, such displays do not fully capture the properties of optic flow generated in the real world. Physical self-motions typically occur in environments consisting of extended/continuous surfaces. The global structure of the optic flow generated by self-motions in such environments will depend on three surface properties: 3-D shape, as well as diffuse and specular reflectance. In this study, we examined the roles that these three surface properties play in the visual perception of self-motion.

The reflectance of most opaque surfaces can be modeled using a bidirectional reflectance distribution function. The intensity of diffuse reflectance depends not only on the albedo of the surface, but also on the angle of incident light relative to the surface normal. Because diffuse reflectance is independent of the observer's vantage point, light is distributed diffusely in all directions around a given surface normal, according to a cosine function (see Fig. 1a). Specular reflectance distributes 
light less broadly over a narrower lobe than does diffuse reflectance. As a result, specular reflections are generated at locations in the image that correspond to surface regions with normals that bisect the angle formed between the illumination and viewing directions to the same surface point. Hence, specular reflections will tend to be less abundant in retinal images than will diffuse shading.

As is shown in Fig. 1b and c, these reflectance properties also have different consequences for the patterns of light reflected by surfaces as an observer moves through the visual environment. The global velocity of the optic flow generated by diffuse reflectance is approximately the inverse of the observer's velocity. In contradistinction, the specular reflectance of a surface is viewpoint-dependent and tends to generate lower velocities of specular (relative to diffuse) flow. This is exemplified in the example of self-motion relative to a stationary point light source shown in Fig. $1 \mathrm{~b}$ and c. The diffusely shaded ground texture is seen to move eccentrically to a larger extent than does the specular highlight. In the case of such flat surfaces, only the optic flow component generated by relative motion of the diffuse texture is informative about the true location of the observer within the environment. These diffuse
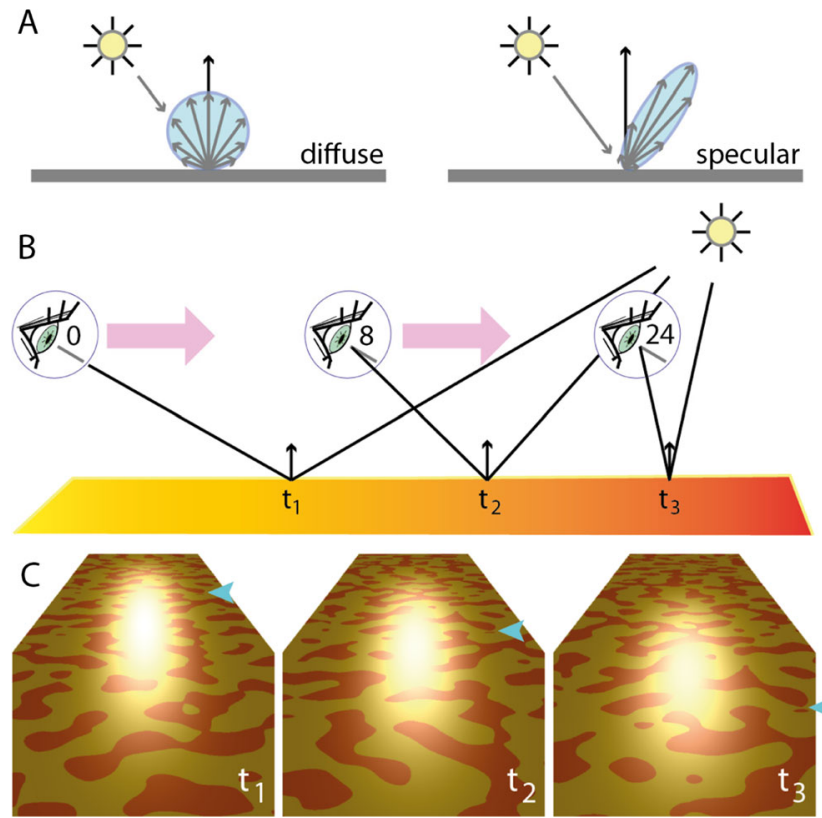

Fig. 1 Optic flow generated by diffuse and specular reflectance during linear self-motion. (A) Schematic showing the difference in the ways that light is distributed around surface normals in diffuse and specular reflectance. (B) Profile showing the specular reflectance of a distal light source into the observer's eye translating forward in depth. Note that over time $\left(t_{1} t_{3} t_{3}\right)$, the optical displacement of a specular reflection away from foveal vision into the periphery is considerably smaller than the displacement of any finite point passed by the observer. (C) Simulations showing the same concept from the perspective of the observer. Between times $t_{1}$ and $t_{2}$, the specular highlights generated by a distant light source move less in the image than do the diffuse points at the same initial location. The arrow on the right of each frame shows the location of the same surface point in the image over time and specular motions appear to be separable on the basis of relative differences in the velocities of the optic flows they generate. However, this velocity cue may only be useful for the special case of optic flow caused by purely flat surfaces.

Most real-world surfaces are not perfectly flat, but tend to be uniform in albedo with 3-D relief and generate highcontrast diffuse and specular components that could (in principle) contribute differentially to vection. Previous studies have shown that vection is highly influenced by the parameters of display size and the density of moving contrasts (Brandt, Wist, \& Dichgans, 1975; Lestienne, Soechting, \& Berthoz, 1977). Brandt et al. found that rotary vection increased as a function of the number of moving display elements. This finding was also replicated for linear vection (Lestienne, Soechting, \& Berthoz, 1977), suggesting that vection generically depends on the density of moving contrasts in an optic flow display. This reported dependence of vection on the density of the optic flow field could underlie any potential effects of different surface properties on vection. For example, surface relief height influences the formations of diffuse and specular contrasts (Marlow, Kim, \& Anderson, 2012): Increasing the relief height of surfaces tends to increase the abundance of diffuse contrasts, but also to reduce the number of specular contrasts. This shows that diffuse and specular optic flow fields share complex interdependencies on 3-D surface relief.

No previous research has systematically explored the potential effects of diffuse and specular optic flow on vection. Riecke et al. (2006) provided some evidence that dynamic scenes rendered with realistic surface properties can improve the experience of vection. Although they found that the simulation of more complex surface properties - including diffuse and specular reflectance - enhanced self-motion perception, some of these enhancements may have been due to increases in the size and contrast of the optic flows created by combining diffuse and specular components. Additionally, information concerning perceived 3-D shape across displays with diffuse and specular reflectance was not determined. Perceived shape has been identified as an important factor in the perception of gloss (Marlow \& Anderson, 2015), and possibly also important for vection. It is therefore unclear whether the increases in perceived self-motion when combining diffuse and specular components are attributable only to variations in the low-level parameters of optic flow, or also to the midlevel recovery of surface properties.

The present study simulated self-motions relative to continuous surfaces to examine whether linear vection is differentially influenced by optic flow generated by diffuse and specular reflectance. The primary aim of this study was to ascertain whether specular optic flow contributes to the perception of self-motion, and the extent to which such percepts might differ from perceived self-motions generated by diffuse optic flow. We considered the roles of three main surface 
attributes in the perception of self-motion: the surface's diffuse reflectance, its specular reflectance, and the perceived surface relief. Experiment 1 compared the vections induced by pure specular flow, pure diffuse flow, and combined specular and diffuse flow. In Experiment 2, we sought to ascertain whether specular highlights are processed independently of diffuse flow or merely contribute to the density of moving contrasts.

\section{Experiment 1}

Most previous studies have used simple computer-generated 3$\mathrm{D}$ cloud (or dot motion) displays to generate optic flow simulating self-motion in depth (see Palmisano et al., 2011, for a review). These displays were not designed to simulate the reflectance properties of natural surfaces. Although a few studies have examined the vection induced by moving surfaces with complex reflectance profiles (e.g., by using computer-generated imagery in Riecke et al., 2006, or real-world image sequences in Bubka \& Bonato, 2010), they were not designed to identify the relative contributions of diffuse and specular shading to selfmotion perception. By contrast, in Experiment 1 we used computer graphics to examine the independent effects of the diffuse and specular optic flow components on vection in depth. We created custom software to generate a ground plane with relief, which allowed us to examine the effects of generating radial optic flow by specular shading only, diffuse shading only, or combined specular and diffuse shading.

Using this software, we performed some pilot test renderings and initial data collection (see the Appendix). We simulated self-motion in depth through a linear tunnel with relief and found that although specular-only flow did generate vection, its strength appeared to be inferior to that induced by diffuse-only and combined optic flows. However, we also noticed that specular highlights tend to be constrained to appear near the central region of the image, which appeared to be caused by the viewpoint dependence of specular reflectance. In contradistinction, diffuse shading was found to generate locally moving contrasts at all eccentricities in the image. Hence, differences in the display size and eccentricity of optic flow must be controlled between specular and diffuse conditions in order to undertake a fair psychophysical comparison of their vection-inducing potentials. In Experiment 1, we controlled eccentricity by introducing multiple light sources situated in depth.

If vection depends on low-level visual motion cues, then we might expect illusory self-motion to be weakest in the specular-only condition, since this condition generates lower-net-velocity retinal motion than does the diffuse-only condition (Andersen \& Braunstein, 1985; Apthorp \& Palmisano, 2014). Combining specular and diffuse flow might also reduce vection relative to the diffuse-only condition (since averaging the two flow components would generate a lower net velocity of retinal motion than diffuse-only flow, although the velocity would still be greater than that in the specular-only condition). To alter the dynamics of radial specular flow, we imposed conditions in which the lighting was stationary relative to the surface and moved relative to the observer (world-fixed) or was fixed relative to the observer and moved relative to the surface (observer-fixed). This was done to determine whether changing the rate of radial specular flow alters vection.

\section{Materials and method}

Observers Six adult observers with normal or corrected-tonormal visual acuity participated in this experiment. All procedures were approved by the Human Research Ethics Advisory (HREA) panel at the University of New South Wales.

Stimuli We generated an artificial 3-D environment in the form of a curved ground plane using the mesh functions provided in OpenGL libraries compiled in a custom application written in Microsoft Visual C++ 2010 Express, running in the Window 8.1 operating environment on a Toshiba Satellite computer with an i5-4200U CPU and an AMD Radeon R7 M260 graphics card. The stimuli were presented on a 21-in. Mitsubishi Diamond Pro 2070SB CRT monitor and viewed at a distance of approximately $45 \mathrm{~cm}$. The display had a viewing range of approximately $49^{\circ}$ horizontal and $37^{\circ}$ vertical, similar to the range in a recent vection study (Kim $\&$ Khuu, 2014). The luminance of the display's black and white points ranged between 0.5 and $320 \mathrm{~cd} / \mathrm{m}^{2}$, though the working range for presenting optic flow was within an upper limit of approximately $40.0 \mathrm{~cd} / \mathrm{m}^{2}$. The background luminance in the periphery was adjusted, using ambient lighting in the specular-only conditions, to a nominal intensity of approximately $10.4 \mathrm{~cd} / \mathrm{m}^{2}$.

The ground plane was initially constructed in triangular strips with vertex positions falling on the circumference of a cylinder oriented in depth. The radius of each vertex in each ring was randomized by $5 \%$ of the radius to produce a rigid tunnel with relief. We then omitted the top half of the cylinder and scaled the radius by $50 \%$ in the vertical direction. This generated a ground-plane terrain with multiscale curvature and bumpiness (see Fig. 2).

We used a finite number of facets (strips constructed from the triangles), distributed in depth to increase the rendering performance. This was similar to the method of a previous study that had simulated self-motion relative to 3 -D clouds of square objects that loomed in depth (Kim \& Palmisano, 2008). All strips and vertex positions moved toward the observer on each frame. When a particular strip moved beyond the observer's viewpoint, it was deleted and a new strip was randomly generated and appended to the far end of the ground 
plane. This approach simulated smooth and continuous motion of the observer relative to a rigid ground-plane terrain that was seemingly unlimited in depth.

We rendered specular-only, diffuse-only, and combined specular and diffuse motion sequences. There were three light sources - separated by regular intervals (approximately every $3.5 \mathrm{~m}$, or half of the simulated distance in depth). This lighting was observer-centered for half of the trials; that is, the lighting moved with the observer. The remaining trials were world-centered and generated motion of the lighting and the terrain relative to the observer. We increased the background luminance of displays containing pure specular flow to be comparable to the displays containing diffuse shading flow.

Procedure Observers were initially familiarized with the apparatus and shown a 3-D cloud optic flow display, similar to those used in typical vection studies (identical to that used in a previous study by Kim, Chung, Nakamura, Palmisano, \& Khuu, 2015). The cloud comprised blue square objects arranged in a spherical space simulated around the observer (approximately $3-\mathrm{m}$ radius and 163,840 objects ranging in optical size from 0.25 to $2.5 \mathrm{deg}$ with proximity to the observer; the luminance of the dots was $3.5 \mathrm{~cd} / \mathrm{m}^{2}$ against a black background of $0.11 \mathrm{~cd} / \mathrm{m}^{2}$ ). Observers were informed that they would be required to stare at the center of similar flow fields and to concentrate on any experience of illusory selfmotion. Following each self-motion display, observers were instructed to adjust a horizontal rating bar, using the arrow keys to report the overall strength of the vection they experienced for the trial. The rating scale ranged between 0 and 100 $(0=$ completely stationary the whole time,, $100=$ experienced self-motion indistinguishable from physical self-motion the whole time). Pressing the spacebar recorded the vection strength rating and commenced a 3-s delay in total darkness prior to the presentation of the subsequent trial. The motion display phase of each vection trial was $30 \mathrm{~s}$ in duration. Visual motion with two lighting conditions and three reflectance conditions was randomly presented in each 30 -s trial $(2 \times 3)$. Each observer performed at least two repeated blocks in a single experimental session lasting approximately $20 \mathrm{~min}$.

Data analysis The vection strength ratings were recorded to an ASCII file following each trial. We analyzed the vection strength data using a two-way repeated measures analysis of variance (ANOVA). We also computed local and global rootmean square (RMS) contrast to determine the net number of moving contrasts in the three different display types.

\section{Results and discussion}

Figure 3 shows the mean vection strength ratings for each of the three simulated reflectance conditions. The vection induced under different lighting conditions is represented by separate colored bars, with world-fixed lighting shown in gray and observer-fixed lighting shown in blue. As can be seen, the vection strength responses were fairly uniform across all conditions. A two-way ANOVA of the vection strength data revealed no main effect of surface reflectance $[F(2,10)=0.34$, $p=.72]$ and no main effect of lighting condition $[F(1,5)=$ $0.56, p=.49]$. We also found no significant interaction effect $[F(2,10)=1.20, p=.34]$. World-fixed and observer-fixed lighting generated similar vection, despite marked variations in the speeds of the specular flow components in the different conditions. Thus, it appears that the lighting was sufficient to induce compelling vection (even with specular flow alone), irrespective of its movement relative to the observer.

We also found that the vection induced by specular flow was as compelling as that induced by either diffuse flow or

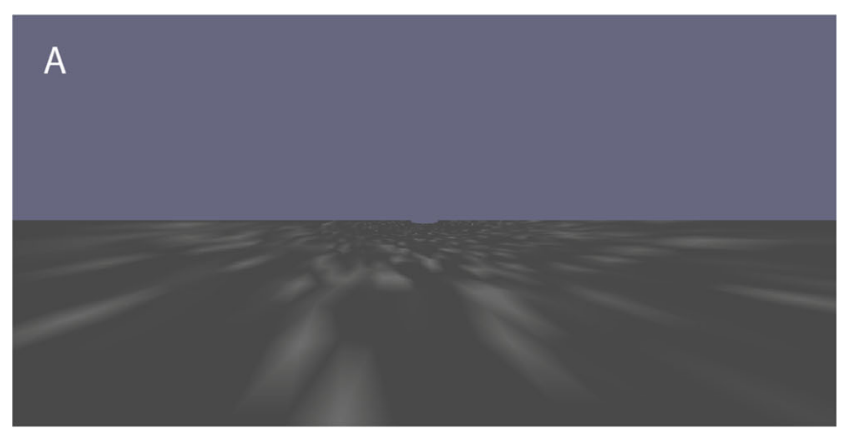

B

C

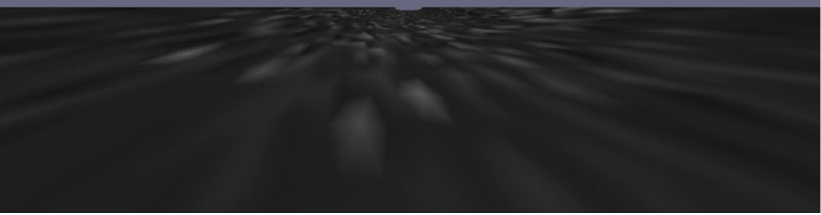

Fig. 2 Layout of the flow fields in the three main conditions: a Specular flow only. b Diffuse flow only. c Combined flow generated by adding both diffuse and specular components together. Note that three light sources were situated at equal intervals in depth, and that they moved relative to the observer (i.e., were world-fixed) on half of the trials. Note that the local contrast of the specular highlights is lower in the specular-only conditions (A) than in the combined (diffuse + specular) conditions (C) 
combined flow. The uniformity in vection responses across these conditions cannot be explained by similarity in the image contrasts. In support of this view, we computed the local and global display contrasts across conditions, the means of which are shown in Fig. 4. A one-way ANOVA showed a significant main effect of display type on global image contrast $[F(2,12)=124, p<.00001]$. However, despite these large differences in global contrast (Fig. 4b), the vection responses for the same conditions were statistically invariant. Additionally, we found no difference in vection between the world-fixed and observer-fixed lighting conditions in which the rate of specular flow was varied. Rather than simply depending on the motion of contrasts per se, vection may have also depended on the distribution of these moving contrasts. The improved vection generated by specular flow in this experiment (as compared to that induced by specular flow in our pilot study — see the Appendix) could potentially be explained by the similarity in eccentricity of the flows generated by both specular and diffuse components (Fig. 4a). In the next experiment, we attempted to determine whether vection depends on independent processing of specular and diffuse flows following an initial source separation, or on their low-level motion signals.

\section{Experiment 2}

Experiment 1 showed that vection can be induced by specular flow alone. Indeed, under fair conditions, the vection induced by specular flow appears to have a strength comparable to that generated by diffuse flow. Vection was similar across the different lighting/reflectance conditions, even though the RMS contrast varied significantly. Therefore, it is possible that vection may not depend simply on the motion of the display elements per se, but rather on information about the motion of perceived surface "bumps" relative to the observer. This would involve midlevel visual inference of the motion of rigid surface curvatures, rather than simply the motion of edge contrasts across the retina. This is feasible, given that the perception of 3-D surface relief depends on both diffuse and specular shading (Mooney \& Anderson, 2014). Indeed, the structure of specular shading is known to enhance the perceived relief of surfaces (Norman, Todd, \& Orban, 2004). Therefore, it is possible that the inferred movement of relief and not of contrasts is important for vection.

It is likely that image highlights must first be attributed to specular reflectance prior to computing relief from their optic flow. Previous studies have shown strong dependencies of perceived shape and gloss on the structure of specular reflections; even small manipulations of specular shading have been shown to eliminate the perception of gloss (e.g., Anderson \& Kim, 2009; Beck \& Prazdny, 1981; Kim, Marlow, \& Anderson, 2011; Todd, Norman, \& Mingolla, 2004). For example, specular highlights are generated at regions of brighter diffuse shading, and displacing them into darker regions decreases the perceived gloss (Kim et al., 2011). In a similar way, inverting the luminance of the specular highlights in an image eliminates the perceived gloss (Marlow \& Anderson, 2013). In Experiment 2, we performed similar luminance inversions of specular highlights, which should prevent the attribution of these image contrasts to specular reflections. This approach ensured that the image contrast did not change; all of the underlying spatial frequency distributions and net motion energies were roughly preserved because we were altering the local sign of the specular contrasts, but not the pattern of the edges they generated. This manipulation should eliminate the experience of surface relief. If successful, one might predict that the decline in perceived relief should generate weaker vection than in conditions in which the specular reflections were physically correct.

\section{Materials and method}

Observers Eight adult observers with normal or corrected-tonormal visual acuity participated in this experiment. All procedures were approved by the HREA panel at the University of New South Wales.

Stimuli The image sequences were prerendered using Blender 3D. This was done to increase the quality of the renderings to be close to photo-realistic. Each grid, composed of 396,294 vertices, was initially deformed using a pink-noise height map. The camera was oriented to view the surface frontally so that $90 \%$ of the surface filled the field of view. A single collimated light source was used to illuminate the surface plane frontally, but it was angled slightly from above by $15 \mathrm{deg}$. This ensured that the specular flow conditions generated specular highlights that had a spatial distribution across the image that was similar in eccentricity to the visual elements generated in the diffuseonly flow conditions.

Figure 5 shows sample image frames for each of the rendering conditions. The images in the top row are raw renderings with diffuse shading only, specular shading only, or both in additive combination. The lower row shows images in which the luminance profile is inverted. To ensure that the highlights would be maximally incompatible with the adjacent shading in the combined case, we only inverted the luminance of the specular component relative the original diffuse shading profile (i.e., we did not invert the luminance of the entire image). For the diffuse-only conditions, vivid surfaces with 3-D relief were experienced in either the original or the inverted-polarity images. For the conditions with specular highlights, the surfaces appeared as glossy surfaces with relief in the original renderings. However, the inverted specular highlights appeared very differently and did not generate vivid experiences of 3-D surfaces. 


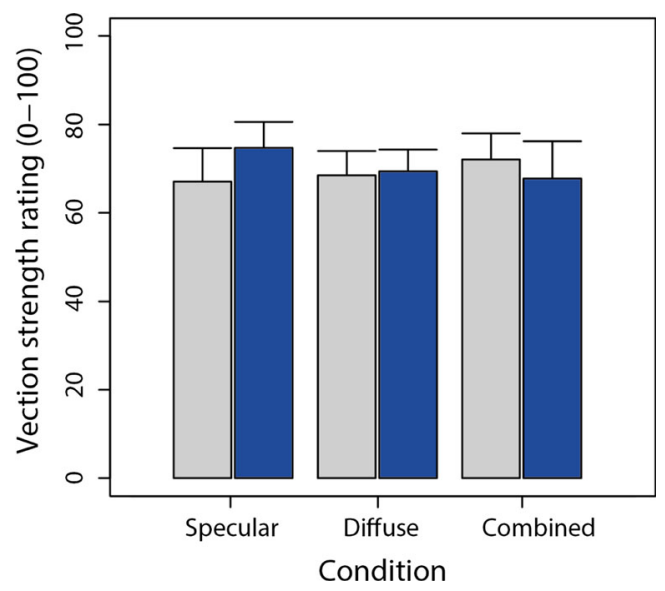

Fig. 3 Mean vection strength ratings for different lighting and reflectance conditions: Means computed directly from the vection strength ratings across all observers for each condition, with specular shading only, diffuse shading only, or both diffuse and specular components combined. Vection strength for world-fixed lighting is shown in light gray, whereas vection for observer-fixed lighting is in blue/dark gray. Error bars are standard errors of the means

Procedure We compared the strengths of the vection induced by the vertical motion of the surfaces relative to the observer. Movie sequences of vertical flow were presented in order to visually simulate downward self-motion. The observer experienced 30-s trials with two repeats for all six test conditions (12 trials in total). All conditions were randomized within each block of repeat trials. A delay of approximately $10 \mathrm{~s}$ was provided between trials, to allow observers to prepare for the subsequent trial. Observers indicated their vection strength experiences for each trial using the same rating scale as in Experiment 1.

At the end of the vection testing, observers were shown short, 8-s presentations of each flow condition once more, again in random order. They were instructed to rate the "overall bumpiness of the surfaces" they saw in these moving images. The same rating bar was used to record their perceived relief height estimates, which ranged from 0 (completely flat) to 1 (bumpy like raw granite). The responses were recorded and averaged.

Data analysis We analyzed the vection strength and perceived relief height data using two-way repeated measures ANOVAs. Bonferroni-corrected $t$ tests were used in which pairwise planned comparisons were required. A Pearson's productmoment correlation was used to test for any relationship between vection strength and perceived relief height.

\section{Results and discussion}

Figure 6 shows bar charts for vection strength and perceived relief height for each of the three flow conditions (matte/diffuse, specular, and combined). Separate colors show responses to different specular and diffuse polarities: the
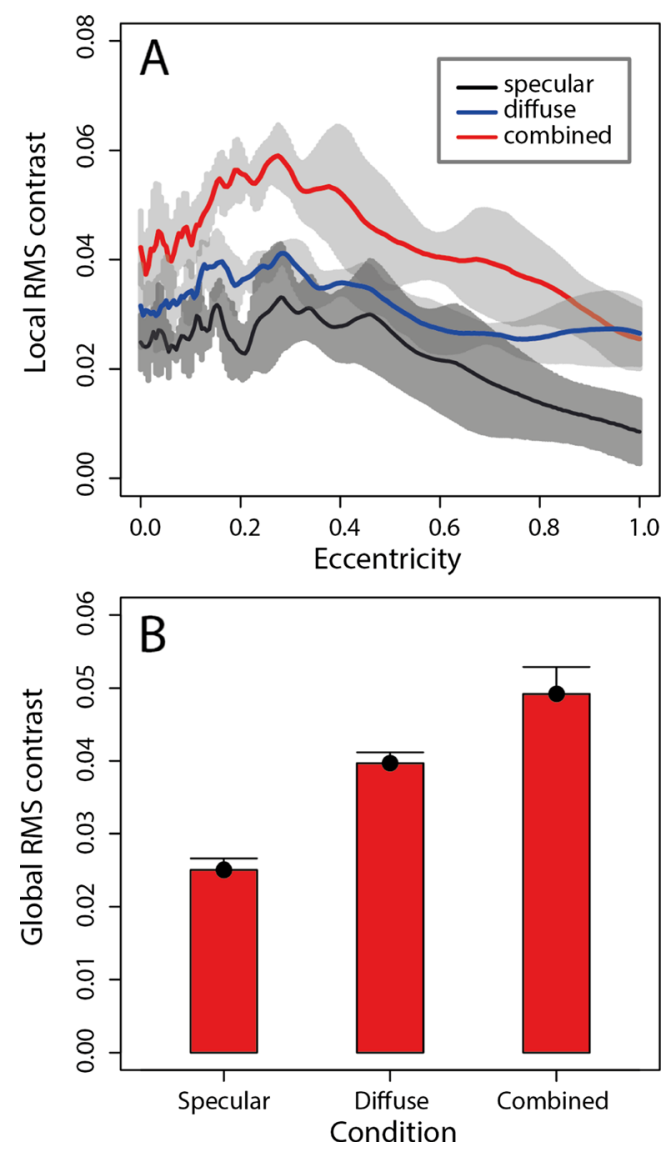

Fig. 4 Root-mean square (RMS) contrasts for the ground plane. (A) Local RMS contrast plotted as a function of eccentricity, defined as vertical location in the image from the center. Separate curves show the mean local contrast computed over five randomly selected frames from the image sequences from each condition. Note that the contrast spans the full eccentricity of the display in all conditions. (B) Global RMS contrasts for the same images. Image contrast increases between the specular and diffuse displays, and combining specular and diffuse components generates displays with the greatest overall contrast. Error bands are standard errors of the means

original renderings (light) and negatives of the originals (dark). For the vection strength data, a two-way ANOVA showed a significant main effect of rendering condition on the vection strength $[F(2,14)=10.33, p<.005]$. We found no significant main effect of image polarity $[F(1,7)=0.82, p=.78]$, but there was a significant interaction effect of both rendering condition and image polarity on vection strength $[F(2,14)=4.68, p<.05]$. Bonferronicorrected planned contrasts revealed a significant difference in vection between the displays with inverted specular reflections and those with the original-polarity specular highlights $(p<.05)$. No significant difference in vection emerged between the two different polarities for purely diffuse shading flow $(p>.05)$.

For the perceived relief height data, a two-way ANOVA revealed a significant main effect of rendering condition on perceived relief height $[F(2,14)=10.33, p<.005]$. There was 
no significant main effect of image polarity on perceived relief height $[F(1,7)=0.20, p=.62]$. However, we did observe a significant interaction effect of both rendering condition and image polarity on perceived relief $[F(2,14)=16.22, p<$ $.0005]$. Bonferroni-corrected planned contrasts showed a significant difference in perceived relief height between displays with inverted specular reflections versus the original-polarity specular highlights $(p<.05)$. No significant difference in perceived relief was apparent between the two different polarities of purely diffuse shading flow $(p>.05)$.

Given the similarity in the patterns of data between vection and perceived relief height, we investigated this relationship further. Figure 7 plots normalized vection strength as a function of perceived relief height across all observers. We found a significant positive correlation between normalized vection strength and the perceived relief height of the surfaces viewed $[r=.50 ; t(46)=3.87, p<.0005]$. These results together indicate that vection is moderately correlated with the perceived relief of surfaces. These results are consistent with the view that perceived relief depends on the appropriateness in the structure of specular reflections, relative to diffuse shading. Inverting the profile of diffuse shading did not significantly alter the perceived relief height (and subsequent vection), due to the bas relief ambiguity. The possibility that vection depends on perceived relief is discussed further below in the General Discussion.

\section{General discussion}

We initially sought to determine whether specular optic flow is sufficient to generate compelling visual experiences of self-motion perception. We simulated self-motion relative to continuous surfaces and measured the strength of the vection that different displays induced. Experiment $1 \mathrm{dem}-$ onstrated that specular flow alone was indeed sufficient to generate vection. Under the specific stimulus conditions of this experiment, the vection induced by specular-only flow was comparable to that induced by diffuse-only flow, and combining diffuse and specular components together did not significantly alter the vection strength. Despite the uniformity in vection across diffuse and specular shading conditions, we found that the RMS contrast varied considerably across these conditions. In Experiment 2, we found that inverting the luminance of specular highlights reduced vection strength, and this decline was correlated with perceived relief height. These findings together support the view that vection does depend on computations involved in the perception of surfaces and materials, rather than simply on low-level mechanisms.

The addition of the specular component to diffuse shading in Experiment 1 increased the contrast of moving display elements (and possibly also their density), but a contrast-only explanation of the vection results does not appear valid. The combination of specular and diffuse components in Experiment 1 did not increase vection beyond that obtained with purely diffuse displays, suggesting that processing beyond low-level motion is important for vection. This is also supported by the finding that vection did not vary between conditions with observer-fixed and world-fixed lighting, in which specular radial flow was varied. Hence, despite these differences in overall image contrast and motion dynamics, we found no significant differences in vection strength across all lighting and reflectance conditions that we tested (Exp. 1).

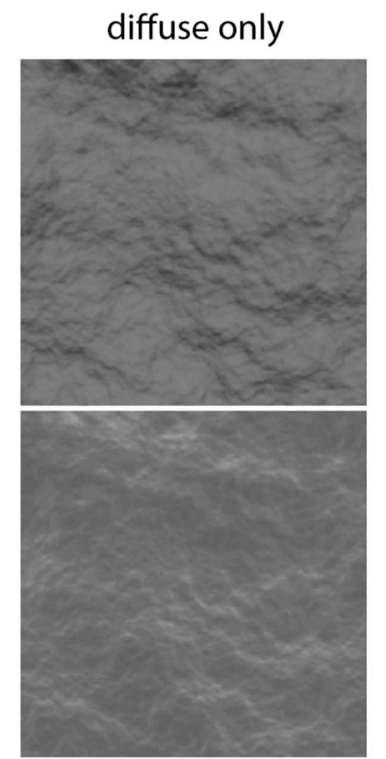

Fig. 5 Sample image frames for each of the rendering conditions. (Top row) Raw renderings with diffuse shading only, specular shading only, or both in combination (diffuse + specular). (Lower row) Same images, but

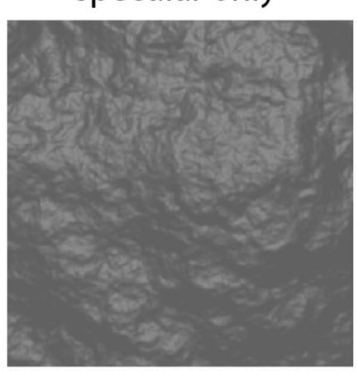

\section{diffuse+specular}

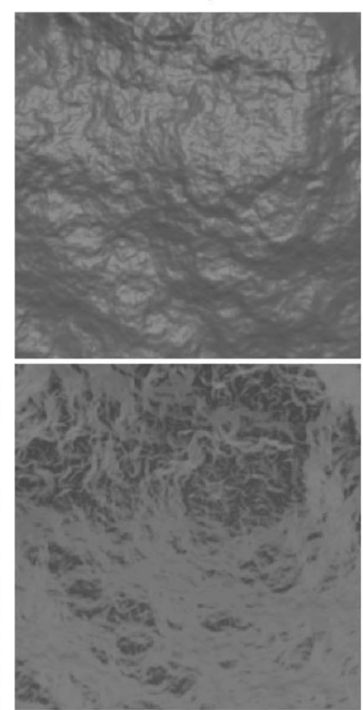

with the luminance profiles inverted. Note that only the specular highlights were inverted in the combined case, relative to the original diffuse component 
We also found that vection was correlated with the perceived relief height of surfaces (Exp. 2), and that destroying the physical correctness of specular highlights reduced perceived relief height. It is possible this decline might explain the associated decline in vection strength. If true, this would support the view that vection depends (at least in part) on the computation of moving surface curvatures, and not merely on moving image contrasts. Previous studies have shown that the attribution of image highlights to specular reflectance depends on their luminance profile relative to surrounding shading (Anderson \& Kim, 2009; Beck \& Prazdny, 1981; Kim, Marlow, \& Anderson, 2011, 2012; Marlow \& Anderson, 2013; Todd, Norman, \& Mingolla, 2004). In particular, Marlow and Anderson (2013) found that inverting the luminance profile of specular reflections decreased the experience of surface gloss. Similar declines were apparent in the present study, which were also accompanied by significant declines in
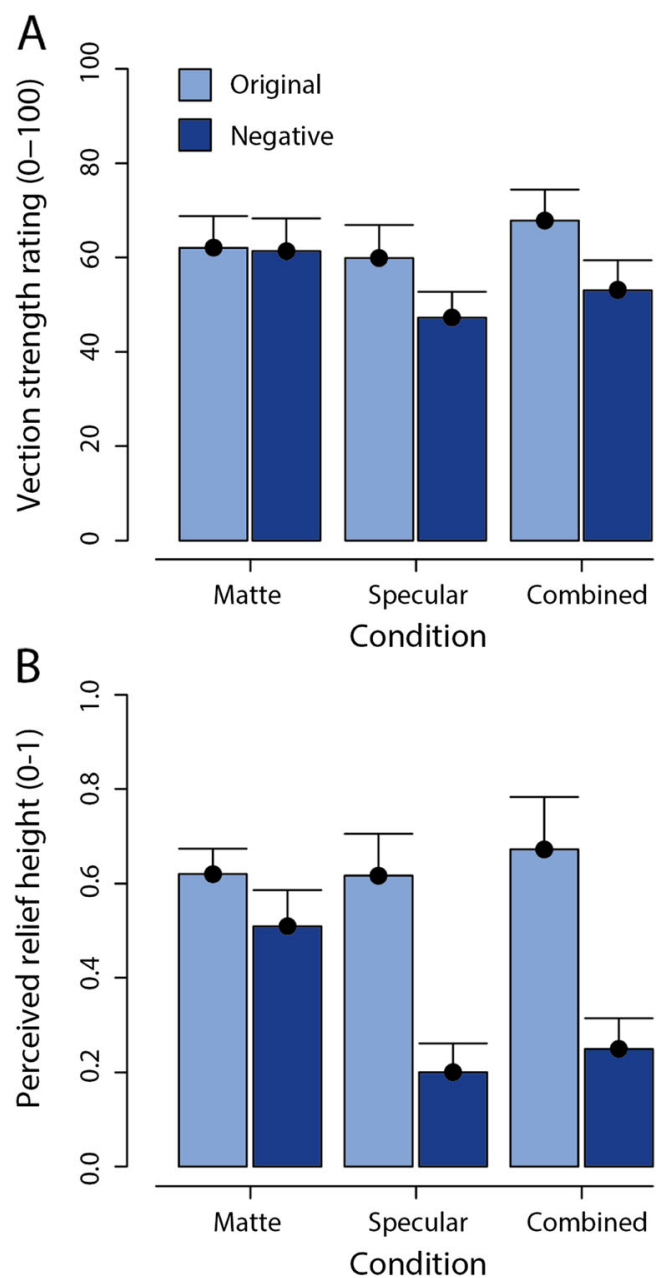

Fig. 6 Psychophysical results of Experiment 2. (A) Mean vection strength ratings for conditions with diffuse shading only (matte), specular shading only, or both specular and diffuse shading combined. Separate bars show the responses to raw renderings (lighter bars) and to renderings with luminance inverted (darker bars). (B) Mean perceived relief height for the same conditions. Error bars are standard errors of the means

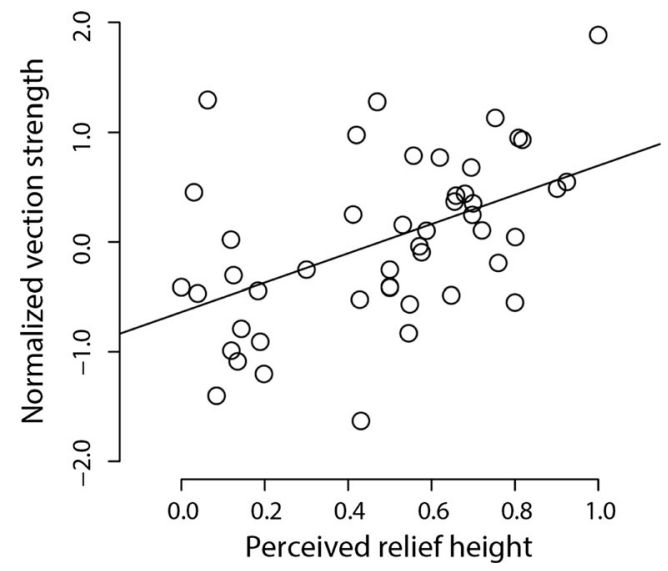

Fig. 7 Normalized vection strength estimates plotted as a function of perceived relief height. The line of best fit is superimposed. Values for perceived relief height range from 0.0 (completely flat) to 1.0 (very bumpy). Note the positive relationship between vection strength and perceived surface relief height $(r=+.49)$

perceived relief height. We propose that the accompanying decline in vection strength can be explained by the decline in perceived relief, which points to independent streams of the visual motion processing of surface relief from specular and diffuse shading flow. Thus, the relative enhancement of vection in the specular-only conditions of Experiments 2 would appear to be best explained by visual computation of relief motion, and not by moving image contrasts per se.

Diffuse shading also appears to undergo midlevel processing, even though we found no effect of luminance inversion on either the vection or perceived relief generated by purely diffuse flow. It is well known that the perceived relief height of planar surfaces generates a bas relief ambiguity, whereby the experience of vivid relief in surfaces is generated by either upright or inverted images. The interpretation of relief tends to depend on an assumed light source from above, and it has been shown to modulate midlevel estimates of surface properties (e.g., perceived lightness; see Kim et al., 2014). The

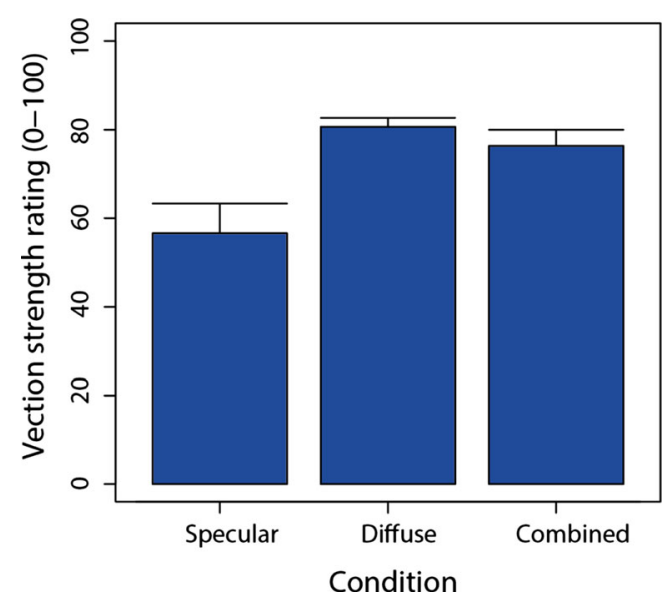

Fig. 8 Mean vection strength ratings for the three reflectance conditions (specular, diffuse, and combined). Error bars are standard errors of the means 
absence of any effect of inversion of uniform-albedo surfaces on perceived relief height and vection in our study is consistent with this level of visual processing.

Previously, no studies had explicitly examined the effects of different surface optics on self-motion perception, but some researchers have considered the effects of lighting on vection induced by the motion of noncontinuous surfaces. Nakamura et al. (2013) examined whether lighting could modulate the perception of self-motion generated by displays consisting of looming square objects. Their 3-D cloud displays simulated self-motion in depth (i.e., radial optic flow). The lateral position of the light source was oscillated about the center of the display to generate horizontal second-order transparent motion, based on the changing photometric energy reflected by the objects on either side of the display. They found that these dynamic orthogonal lighting oscillations reduced the strength of vection in depth, compared with conditions when the
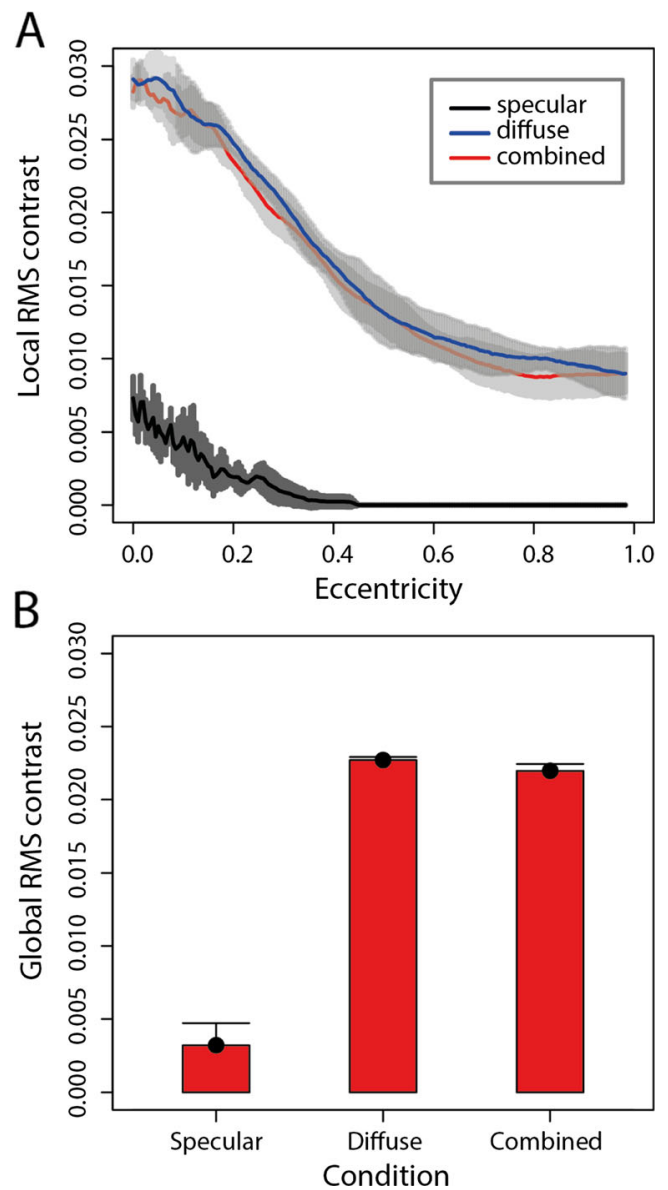

Fig. 9 Root-mean square (RMS) contrasts for tunnel displays. (Top) Local RMS contrast computed over each row of pixels at different magnitudes of eccentricity, ranging from the center of the display (0.0) to the lowermost edge of the display (1.0). Means were computed over five randomly selected frames from the image sequences for each condition. (Bottom) Global RMS contrasts computed over the entire image for the same images. Image contrast increased between the specular and both the diffuse and the combined displays. Error bands and bars show the standard deviations of the means lighting position remained static. An earlier study showed that dynamic chromatic and achromatic modulation of the flow field also affected the strength of vection in depth (Nakamura et al., 2010). The findings from these previous studies suggest that the experience of self-motion is sensitive to source contamination of the optic flow field due to dynamic lighting properties. However, we did not find such effects in Experiment 1 when lighting moved collinearly in the same direction as the observer; there was no difference between the effects of observer-fixed and world-fixed lighting on vection.

The findings obtained here with simulated surfaces offer new perspectives for interpreting the ecological characteristics of conventional vection stimuli, typically composed of random-dot or random-object motion displays. One recent study by Ogawa et al. (2014) did generate optic flow by displacing groups of identical tiles textured with images of static scenes (nine different tile types were examined, each depicting a single surface rendered with different material 'qualities', including glossiness). Although Ogawa and colleagues found that the group displacement of such images could induce compelling vection, the strength of this vection did not appear to depend on the material qualities of the surfaces rendered in these images. However, it is important to note that the surface properties of the tile images themselves did not contribute to the optic flow field, as they normally would during their real viewpoint-dependent displacement (i.e., the structure of luminance variations in the tile images did not change over time). This particular limitation of that study prevented a specific analysis of the roles of diffuse and specular flow properties in the perception of self-motion.

In contradistinction, we found here that surface optics do contribute differentially to vection, and that specular flow alone is sufficient to generate compelling visual experiences of self-motion perception. The findings in previous research suggest that perceived speed and vection are correlated (e.g., Kim \& Palmisano, 2008). It is possible that the computation of surface relief might differentially affect perceived speed, which could be explored in the future. It may also be worthwhile considering the role of the realism of simulated displays (e.g., Riecke et al., 2006) and the relative contributions of textural, specular, and diffuse flows to the vivid experience of both surface properties and self-motion perception.

Author note Many thanks to B. Cheng for technical assistance. This research was funded by an Australian Research Council Future Fellowship awarded to J.K. (FT140100535).

\section{Appendix}

We performed an initial pilot test to verify that specular flow alone can induce vection. This test compared the strengths of the vection induced by specular-only flow, diffuse-only flow, 
and combined flow. We also examined differences in the eccentricities of the moving contrasts generated by the diffuse and specular flow components, in order to determine whether such differences might need to be addressed in the main study.

\section{Materials and method}

Observers Four adult observers with normal or corrected-tonormal visual acuity participated in the experiment. All procedures were approved by the HREA panel at the University of New South Wales.

Stimuli The stimuli were similar to those in Experiment 1, except that a complete circular tunnel was rendered on each frame (rather than a ground plane). Relief was introduced using the same randomized vertex displacement as in Experiment 1. Background luminance in the periphery was adjusted using ambient lighting in the specular-only conditions to a nominal intensity of approximately $10.4 \mathrm{~cd} / \mathrm{m}^{2}$. This was done to ensure that the highlights had similar intensity differences relative to the background across specularonly and combined conditions (diffuse + specular).

Procedure The procedures and briefing of observers were the same as in Experiment 1. Observers were instructed to provide vection strength ratings but were not required to report vection onset latencies during the presentation of each trial. In addition to analyzing differences in vection strength using a one-way ANOVA, we computed the RMS image contrast locally along each row of pixels in frames sampled from each display condition. We also computed contrast globally across all pixels in the image. This allowed us to determine whether any potential image properties could account for the vection strength responses.

\section{Results}

The bar graphs in Fig. 8 show the means and standard errors for the vection strength ratings across the three conditions with different simulated reflectance properties. A one-way ANOVA revealed a main effect of display type $[F(2,6)=$ $7.11, p<.05]$. Post-hoc $t$ tests showed that raw vection was significantly stronger in the diffuse-only shading condition than in the specular-only shading condition $[t(3)=$ $4.01, p<.05]$, and that combining both diffuse and specular components did not significantly alter the raw vection strength relative to the vection generated by diffuse shading alone $[t(3)=1.06, p=.37]$.

Figure 9 shows the RMS image contrast of the different display conditions, computed locally as a function of eccentricity and globally across the entire image. Although the luminance-defined contrasts spread all the way from the center of the display (0) out to its farthest edges (1.0) in the conditions with diffuse-only or combined shading, the spread was considerably narrower in the specular-only conditions (specular cutoff values: $M=.37, S D=.07$ ). Cutoff eccentricity values were significantly smaller for the specular-only conditions $(M=.37, S D=.07)$ than for the other display conditions $[t(4)=18.81, p<.00001]$. Additionally, a one-way ANOVA revealed a main effect of display type on global RMS contrast $[F(2,12)=713.3, p<.0001]$

The vection results cannot be explained by differences in the local contrast of moving elements between the displays, as we increased the background luminance in the specular-only conditions to approximate the contrast in the combined reflectance conditions. However, it is possible that the vection data could be explained by either the relatively low eccentricity or global contrast of specular flow, as compared with the displays containing diffuse-only or combined shading.

\section{References}

Andersen, G. J., \& Braunstein, M. L. (1985). Induced self-motion in central vision. Journal of Experimental Psychology: Human Perception and Performance, 11, 122-132. doi:10.1037/00961523.11.2.122

Apthorp, D., \& Palmisano, S. (2014). The role of perceived speed in vection: Does perceived speed modulate the jitter and oscillation advantages? PLOS ONE, 9(e92260), 1-14. doi:10.1371/journal. pone. 0092260

Anderson, B. L., \& Kim, J. (2009). Image statistics do not explain the perception of gloss and lightness. Journal of Vision, 9(11), 10, 1-17.

Beck, J., \& Prazdny, S. (1981). Highlights and the perception of glossiness. Perception \& Psychophysics, 30, 407-410.

Brandt, T., Wist, E. R., \& Dichgans, J. (1975). Foreground and background in dynamic spatial orientation. Perception \& Psychophysics, 17, 497-503.

Bubka, A., \& Bonato, F. (2010). Natural visual-field features enhance vection. Perception, 39, 627-635. doi:10.1068/p6315

Dichgans, J., \& Brandt, T. (1978). Visual-vestibular interaction: Effects on self-motion perception and postural control. In R. Held, H. Leibowitz, \& H. L. Teuber (Eds.), Handbook of sensory physiology: Vol. 8. Perception (Vol. 8, pp. 755-804). New York, NY: Springer.

Gibson, J. J. (1966). The senses considered as perceptual systems. Boston, MA: Houghton Mifflin.

Kim, J., Chung, C. Y. L., Nakamura, S., Palmisano, S., \& Khuu, S. K. (2015). The Oculus Rift: A cost-effective tool for studying visualvestibular interactions in self-motion perception. Frontiers in Psychology, 6, 248. doi:10.3389/fpsyg.2015.00248

Kim, J., \& Khuu, S. (2014). A new spin on vection in depth. Journal of Vision, 14(5), 5. doi:10.1167/14.5.5

Kim, J., Marlow, P., \& Anderson, B. L. (2011). The perception of gloss depends on highlight congruence with surface shading. Journal of Vision, 11(9), 4. doi:10.1167/11.9.4

Kim, J., Marlow, P. J., \& Anderson, B. A. (2012). The dark side of gloss. Nature Neuroscience, 15(11), 1590-1595.

Kim, J., Marlow, P. J., \& Anderson, B. L. (2014). Texture-shading flow interactions and perceived reflectance. Journal of Vision, 14(7), 1. doi: $10.1167 / 14.7 .1$

Kim, J., \& Palmisano, S. (2008). Effects of active and passive viewpoint jitter on vection in depth. Brain Research Bulletin, 77, 335-342. 
Lappe, M., Bremmer, F., \& van den Berg, A. V. (1999). Perception of self-motion from visual flow. Trends in Cognitive Sciences, 3, 329336. doi:10.1016/S1364-6613(99)01364-9

Lestienne, F., Soechting, J., \& Berthoz, A. (1977). Postural readjustments induced by linear motion of visual scenes. Experimental Brain Research, 28, 363-384.

Marlow, P. J., \& Anderson, B. L. (2013). Generative constraints on image cues for perceived gloss. Journal of Vision, 13(14), 2. doi:10.1167/ 13.14.2

Marlow, P. J., \& Anderson, B. L. (2015). Material properties derived from three-dimensional shape representations. Vision Research, 115(Part B), 199-208. doi:10.1016/j.visres.2015.05.003

Marlow, P. J., Kim, J., \& Anderson, B. L. (2012). The perception and misperception of specular surface reflectance. Current Biology, 22, 1909-1913. doi:10.1016/j.cub.2012.08.009

Mooney, S. W. J., \& Anderson, B. L. (2014). Specular image structure modulates the perception of three-dimensional shape. Current Biology, 24, 2737-2742.

Nakamura, S., Seno, T., Ito, H., \& Sunaga, S. (2010). Coherent modulation of stimulus colour can affect visually induced self-motion perception. Perception, 39, 1579-1590.

Nakamura, S., Seno, T., Ito, H., \& Sunaga, S. (2013). Effects of dynamic luminance modulation on visually induced self-motion perception:
Observers' perception of illumination is important in perceiving self-motion. Perception, 42, 153-162. doi:10.1068/p7321

Norman, J. F., Todd, J. T., \& Orban, G. A. (2004). Perception of threedimensional shape from specular highlights, deformations of shading, and other types of visual information. Psychological Science, 15, 565-570. doi:10.1111/j.0956-7976.2004.00720.x

Ogawa, M., Hiramatsu, C., \& Seno, T. (2014). Surface qualities have little effect on vection strength. Frontiers in Psychology, 5, 610. doi:10. 3389/fpsyg.2014.00610

Palmisano, S., Allison, R. S., Kim, J., \& Bonato, F. (2011). Simulated viewpoint jitter shakes sensory conflict accounts of self-motion perception. Seeing and Perceiving, 24, 173-200.

Palmisano, S., Allison, R. S., Schira, M. M., \& Barry, R. J. (2015). Future challenges for vection research: Definitions, functional significance, measures and neural bases. Frontiers in Psychology, 6, 193. doi:10. 3389/fpsyg.2015.00193

Riecke, B. E., Schulte-Pelkum, J., Avraamides, M. N., Heyde, M. V. D., \& Bülthoff, H. H. (2006). Cognitive factors can influence selfmotion perception (vection) in virtual reality. ACM Transaction in Applied Perception, 3, 194-216.

Todd, J. T., Norman, J. F., \& Mingolla, E. (2004). Lightness constancy in the presence of specular highlights. Psychological Science, 15, 3339. 